\title{
Factores asociados a la utilización de servicios de salud odontológicos
}

\author{
Factors associated with the use of dental health services
}

Abstract This paper seeks to analyze the factors associated with the use of dental health services (UDHS) by adults in the city of Corrientes, Argentina. A cross-sectional study was conducted. Information concerning the study variables was collected via a home survey. The sample size was established with a 95\% confidence interval level (381 individuals). A simple random sampling design was used, which was complemented with a non-probability quota sampling. The data was analyzed using SPSS version 21.0 and Epidat version 3.1 softwares. Socio-economic level, dental health coverage, perception of oral health care, perception of oral health, knowledge about oral health, and oral hygiene habits were significantly associated with the UDHS over the last twelve months. These same factors, excluding dental health coverage and knowledge about oral health, were associated with the UDHS for routine dental check-ups. Measures should be implemented to increase the UDHS for prevention purposes in men and women of all socio-economic levels, particularly in less-privileged individuals.

Key words Oral health, Dental care, Health services
Resumen El presente trabajo se propone analizar los factores asociados a la utilización de servicios de salud odontológicos (USSO) en individuos adultos de la Ciudad de Corrientes; Argentina. Se realizó un estudio transversal. A través de una encuesta domiciliaria se recolectó información de las variables de estudio. Se determinó el tamaño de la muestra estableciéndose un nivel de confianza del 95\% (381 individuos). Se aplicó un diseño muestral aleatorio simple, que se complementó con un muestreo no probabilístico por cuotas. Se analizaron los datos con los programas SPSS 21.0. y Epidat 3.1. El nivel socioeconómico, la cobertura social odontológica, la percepción sobre la salud bucal, los conocimientos de salud bucal y los hábitos de higiene bucal se asociaron significativamente a la USSO en los últimos 12 meses. Los mismos factores, a excepción de la cobertura social odontológica y de los conocimientos de salud bucal, se asocian a la USSO por motivos de control/ chequeo dental de rutina. Se deben implementar medidas tendientes a aumentar la USSO con fines de prevención en individuos de ambos sexos, de todos los niveles socioeconómicos, especialmente en aquellos menos favorecidos.

Palabras clave Salud bucal, Atención odontológica, Servicios de salud 


\section{Introducción}

Las enfermedades bucodentales están asociadas al estilo de vida de cada persona, y su prevención depende de la adopción de estilos de vida saludables $^{1}$. Los comportamientos importantes de salud bucal que han demostrado tener un impacto positivo incluyen el cepillado de dientes con pasta dental con flúor, la utilización diaria del hilo dental y la asistencia a la consulta odontológica periódicamente para un examen bucodental de rutina ${ }^{2}$.

Existen una variedad de factores que explican las diferencias individuales en la propensión a asumir conductas de salud bucal, entre los mismos se encuentran factores demográficos, sociales, emocionales y de personalidad, así como los factores cognitivos ${ }^{3}$.

Se ha observado que la utilización de servicios de salud odontológicos se ve afectada por aspectos como: edad, sexo, educación, ingreso, ocupación, estatus socioeconómico, disponibilidad de servicios, ubicación geográfica, aspectos culturales, actitudes frente a la salud, valores, estilos de vida, experiencias previas de atención y presencia o ausencia de síntomas, entre otros ${ }^{4,5}$. La utilización de los servicios de salud, desde una perspectiva amplia, implica la necesidad de atención ${ }^{6}$.

Uno de los aspectos importantes al valorar la utilización de los servicios de salud odontológicos es conocer las características del sistema de Salud. Los sistemas de salud deben trabajar en promover la equidad en salud, pero a pesar de ello, por sus lógicas, por sus intereses o por su historia, en determinados contextos, los mismos sistemas de salud profundizan y refuerzan las inequidades en salud ${ }^{7}$. Estudios realizados en países desarrollados señalan la estrecha relación entre las características del sistema de salud y la utilización de los servicios de salud odontológi$\cos ^{8,9}$. En Argentina el Sistema de Salud está compuesto por tres sectores poco integrados entre sí y fragmentados también en su interior: el sector público, el sector de seguro social obligatorio (Obras Sociales) y el sector privado. Los Servicios de Salud son diferentes según la provincia o la ciudad de la que se trate ${ }^{10}$.

La información sistemática sobre los factores que influyen en la utilización de servicios de salud odontológicos en la población adulta de la Ciudad de Corrientes es necesaria con el fin de apoyar la planificación y evaluación de los servicios de salud y para el diseño, elaboración y aplicación de programas de promoción de la salud y prevención de enfermedades bucodentales.
Este estudio se propone analizar la asociación de distintos factores (sexo, nivel socioeconómico, cobertura social odontológica, percepción sobre la salud bucal, conocimientos de salud bucal y hábitos de higiene bucal) con la utilización de servicios de salud odontológicos.

El concepto de utilización, comprende todo contacto directo (consultas médicas y hospitalizaciones) o indirecto (exámenes preventivos y diagnósticos) con los servicio de salud. Es un proceso resultante de la interacción del individuo que busca la atención y el profesional que lo acompaña dentro de un sistema de salud ${ }^{6}$. Para efectos de esta investigación se considerará la utilización de servicios de salud odontológicos como un dato auto-reportado porlos participantes del estudio que declaren haber realizado una consulta odontológica en el transcurso de 12 meses anteriores a la entrevista.

\section{Materiales y Métodos}

Se realizó un estudio transversal en la Ciudad de Corrientes, capital de la provincia de Corrientes, Argentina. La ciudad está situada entre los paralelos 28 y 29 de latitud sur y los meridianos $58^{\circ}$ y $59^{\circ}$ de longitud Oeste. Se halla ubicada sobre el margen izquierdo del río Paraná, con una población urbana aproximada de 350.000 habitantes que constituye una cifra cercana al $40 \%$ del total provincial ${ }^{11}$.

La unidad de análisis estuvo compuesta por habitantes de 35 a 44 años de edad. Se focalizó en este grupo de edad porque la Organización Mundial de la Salud establece esa franja etaria como el grupo estándar de vigilancia del estado de salud bucodental de los adultos ${ }^{12}$. Se incluyeron en el estudio a aquellos individuos que aceptaron voluntariamente contestar las preguntas del formulario de encuesta y se excluyeron a aquellos que no presentaban capacidades físicas y /o mentales para responder el cuestionario y a aquellos que presentaran patologías que impidieran el autocuidado bucodental.

La metodología consistió en la aplicación de una encuesta domiciliaria especialmente diseñada para recolectar información referente a datos sociodemográficos y a conocimientos, actitudes y hábitos de salud bucodental donde figuran las variables consideradas en el presente estudio. Para el relevamiento de los datos se eligió la técnica de encuestas de observación directa mediante entrevistador "cara a cara". El mismo fue realizado por entrevistadores capacitados al efecto. 
La capacitación incluyó los siguientes aspectos: una presentación general del estudio (la temática de la encuesta y la finalidad de la misma); el procedimiento a seguir para la selección de los entrevistados (se los capacitó en la aplicación de los criterios marcados en el diseño de la muestra para el cumplimiento de las cuotas y la identificación de las unidades de la muestra); instrucción en técnicas para presentarse ante la persona seleccionada y motivarla a participar en el estudio; información pormenorizada sobre el cuestionario (el propósito de cada pregunta, cómo formularla y cómo anotar las respuestas). Se proporcionó a los entrevistadores el cuestionario antes de la sesión de formación, proporcionándoles tiempo para que leyeran y reflexionen sobre el mismo, a efectos de poder plantear las dudas que les hubieran surgido. Además se les proporcionó el mapa del área donde se debían realizar las entrevistas y la matriz con las cuotas de sexo que debían respetar. La capacitación se realizó en dos días y los entrevistadores realizaron un juego de roles para afianzar la práctica de la entrevista.

Para comprobar la adecuación del cuestionario como instrumento de medición para lograr los objetivos del estudio se realizó una prueba piloto antes de su administración definitiva a la muestra seleccionada. La prueba se efectuó a 50 sujetos con características similares a la población de estudio (personas adultas de 35 a 44 años de edad). Para ello se aplicó el mismo diseño muestral que se utilizó en el trabajo de campo.

La prueba piloto permitió identificar la necesidad de reformulación de algunas preguntas del cuestionario, simplificar su redacción, eliminar opciones de respuestas como "Todas las opciones" "Ninguna de las opciones" y agregar a algunos ítems opciones de respuestas como "SÍ" "No"; a fin de mejorar la información que se obtiene de las preguntas, su codificación y el análisis de los datos.

El cuestionario elaborado fue examinado por profesionales, docentes e investigadores universitarios de diversas áreas de la salud y la educación.

Concluido el estudio piloto y reformulado el cuestionario, se llevó a cabo el trabajo de campo, previa determinación del tamaño y del diseño muestral. A partir de los datos publicados por el INDEC $^{13}$, se determinó un universo de 42.242 individuos compuesto por 19.639 hombres y 22.603 mujeres. El tamaño de la muestra se determinó con un nivel de confianza del 95\% y un margen de error de +/-5 para estimar la proporción en esta población finita. La muestra quedó conformada por 381 individuos, distribuidos en 204 (53,5\%) mujeres y 177 (46,5\%) hombres. Se aplicó un diseño muestral aleatorio simple para la selección de las viviendas a encuestar, que se complementó con un muestreo no probabilístico por cuotas para la selección de los individuos a entrevistar. Se numeraron todas las manzanas de la ciudad de acuerdo al mapa utilizado en el censo 2010 proporcionado por la Dirección Provincial de Estadística y Censo y se sortearon con el programa estadístico Epidat 3.1 tantas manzanas como número de individuos se incluyeron en la muestra, de manera tal que se entrevistó a un individuo por manzana para garantizar la mayor cobertura de la varianza de la población. Se seleccionó como vivienda la de numeración más baja en la calle correspondiente al lado norte de la manzana.

La selección de los individuos se realizó mediante un muestreo por cuota en función de la variable sexo en el rango de edad seleccionado (35 a 44 años), de acuerdo a la matriz de cuotas que se le proporcionó a cada encuestador.

En cada vivienda seleccionada, se preguntó si había personas con las características establecidas previamente en las cuotas por sexo consignadas en la matriz, en caso de existir dos personas que se ajustasen a las características seleccionadas, se entrevistó al del cumpleaños más próximo; en caso de que no hubiera personas con dichas características, se sustituyó por la vivienda que se encontraba más próxima a la derecha del entrevistador hasta obtener los casos que respondieran a la matriz.

Se solicitó a los sujetos su conformidad para participar del estudio, después de explicarles los objetivos del mismo y se garantizó el anonimato y confidencialidad de las respuestas.

Se realizó la supervisión del estudio piloto y del trabajo de campo definitivo. Se controló la selección de las unidades muestrales (que las personas entrevistadas se correspondieran con la muestra diseñada) y el proceso de entrevista en el $20 \%$ de la muestra. En forma personal y telefónicamente se repitieron varias preguntas que se hicieron durante la entrevista.

El trabajo de campo se llevó a cabo en el año 2013.

\section{Variables de estudio}

- Sexo: variable dicotómica, se consideró femenino; masculino.

- Cobertura social odontológica: esta variable hace referencia a la cobertura de salud (obra social, sistema privado de medicina prepaga, adhesión a programas y planes estatales) (10), cuyo 
beneficio se extiende a la atención odontológica. La variable comprendió la siguiente pregunta: “¿Ud. presenta cobertura social odontológica?" y las posibles opciones de respuestas: "si" - "no".

- Percepción sobre la salud bucal: se preguntó ¿Cómo considera que se encuentra la salud de su boca? Las opciones de respuesta fueron: 1) Muy buena, 2) Buena, 3) Regular, 4) Mala, 5) Muy mala. Para el análisis estadístico se agruparon las opciones 1 y 2 (individuos con percepción buena), y las opciones 3,4 y 5 (individuos sin percepción buena de su salud bucal).

- Conocimientos de salud bucal: se consideraron específicamente los conocimientos referentes a la consulta periódica al odontólogo como factor que contribuye a prevenir las enfermedades bucales más frecuentes. Esta variable comprendió dos preguntas indicadoras: “¿La consulta periódica al odontólogo puede ayudar a prevenir la caries dental?”, y “La consulta periódica al odontólogo puede ayudar a prevenir la inflamación de las encías?". Las posibles opciones de respuestas consideradas fueron: "si"- "no"

- Hábitos de higiene bucal: se consideró la frecuencia diaria del cepillado dental y la utilización diaria del hilo dental. Se preguntó: “ ¿Cuántas veces al día se cepilla los dientes?” y “¿Utiliza diariamente hilo dental?"

- Utilización de servicios odontológicos: se indagó por la consulta odontológica en los últimos 12 meses y por los motivos de la consulta, específicamente por control de rutina/chequeos dentales. Esta variable comprendió dos preguntas indicadoras: “¿Asistió al odontólogo en los últimos 12 meses?”, ,14, "¿Por qué motivos asiste generalmente al odontólogo?".

- Nivel Socioeconómico (NSE):Para establecer el NSE de los entrevistados se utilizó un índice ya empleado y validado en el ámbito académi$\mathrm{co}^{15}$. En el caso de las amas de casa se adjudicó la categoría ocupacional del jefe de hogar. Los indicadores que se emplean para ubicar a los individuos en cada una de las categorías de educación y de ocupación están especificados en el Cuadro 1. El índice de NSE para cada individuo resulta de la combinación de la sumatoria de los puntos obtenidos en los subíndices de nivel educativo y categoría ocupacional. La sumatoria tiene como valor mínimo 2 puntos y, como máximo, 10 puntos. Estos puntos se reúnen luego en las siguientes categorías: bajo ( 2 a 3 puntos), medio-bajo ( 4 a 5 puntos), medio-medio ( 6 a 7 puntos), medio-alto (8 a 9 puntos), alto (10 puntos).

La confiabilidad del índice se evaluó con el coeficiente Alfa de Cronbach. Se consideraron adecuados valores superiores a $0,8^{16}$.

\section{Análisis de los datos}

Se utilizaron los programas estadísticos SPSS 21.0 y Epidat 3.1. Se calcularon frecuencias absolutas y relativas. Se evaluó la asociación estadística mediante el odds ratio (OR) y la significación estadística con la prueba del $\mathrm{X}^{2}$, se tomó como nivel de significación aceptable un valor de 0,05. Igual nivel se utilizó para la estimación de parámetros mediante intervalo de confianza.

\section{Aspectos Éticos}

El estudio cuenta con el aval del Comité de Ética de Investigación de la Facultad de Odontología de la Universidad Nacional del Nordeste.

\section{Resultados}

El 67,2\% de los encuestados informó que asistieron a la consulta odontológica en los últimos 12 meses. El 27\% declaró que el motivo de la consulta fue por control de rutina/chequeo dental. El análisis descriptivo de las variables de estudio se muestran en la Tabla 1. El análisis de cada uno de los factores estudiados en función de la utilización de servicios de salud odontológicos en los últimos 12 meses mostró resultados significativos para las variables: nivel socioeconómico, cobertura social odontológica, percepción sobre la salud bucal, conocimientos y hábitos de salud bucodental (Tabla 2). Las variables percepción sobre la salud bucal y hábitos de salud bucodental fueron significativas en la utilización de servicios de salud odontológicos por motivos de control de rutina/chequeo dental (Tabla 3).

\section{Discusión}

La provincia de Corrientes presenta uno de los Índices más bajos de Desarrollo Humano del País (Corrientes 0,828 Argentina 0,848$)^{11}$, y uno de los niveles más bajos de cobertura de Salud, el 48\% de la población no cuenta con cobertura de salud, mientras que a Nivel País esa suma se reduce al $36,1 \%{ }^{17}$. El $52 \%$ de la población tiene cobertura social, del cual $39 \%$ corresponde a obra social, el $7 \%$ a prepaga a través de obra social, el $3 \%$ a prepaga solo por contratación y el $4 \%$ se encuentra adherido a programas y planes estatales ${ }^{18}$. Investigaciones epidemiológicas focalizadas ${ }^{15,19,20}$ documentan la alta prevalencia de patologías bucodentales que afectan a individuos de distintos grupos etarios que viven en la Ciudad. 
Cuadro 1. Operacionalización del nivel socioeconómico.

\begin{tabular}{|c|l|l|}
\hline \multicolumn{1}{|c|}{ Puntos } & \multicolumn{1}{|c|}{ Nivel educativo } & \multicolumn{1}{|c|}{ Categoría Ocupacional } \\
\hline Bajo (1 punto) & $\begin{array}{l}\text { No hizo estudios; } \\
\text { Primaria incompleta }\end{array}$ & $\begin{array}{l}\text { Obrero; } \\
\text { Desocupado; } \\
\text { Beneficiario de planes sociales; } \\
\text { Servicio doméstico; } \\
\text { Estudiantes }\end{array}$ \\
\hline Medio-bajo (2 puntos) & $\begin{array}{l}\text { Primaria completa; } \\
\text { Secundaria incompleta }\end{array}$ & $\begin{array}{l}\text { Artesano; } \\
\text { Técnico; } \\
\text { Capataz; } \\
\text { Encargado; } \\
\text { Cuenta propia }\end{array}$ \\
\hline Medio-medio (3 puntos) & $\begin{array}{l}\text { Secundaria completa; } \\
\text { Terciaria incompleta }\end{array}$ & $\begin{array}{l}\text { Empleado } \\
\text { Medio-alto (4 puntos) }\end{array}$ \\
\hline Alto (5 puntos) & Universitaria incompleta & $\begin{array}{l}\text { Jefe; } \\
\text { Profesional independiente }\end{array}$ \\
\hline & Universitaria completa & $\begin{array}{l}\text { Dueño con más de 5 empleados; } \\
\text { Gerente; } \\
\text { Director; } \\
\text { Alto Jefe }\end{array}$ \\
\hline
\end{tabular}

Tabla 1. Análisis descriptivo de las variables de estudio. En porcentajes $(\mathrm{n}=381)$. Individuos de $35 \mathrm{a}$ 44 años. Ciudad de Corrientes; Argentina. 2013.

\begin{tabular}{lr}
\hline Sexo & \\
Femenino & 53,5 \\
Masculino & 46,5 \\
Nivel Socioeconómico & \\
Bajo & 15,7 \\
Medio-bajo & 36,7 \\
Medio-medio & 35,2 \\
Medio-alto & 10,8 \\
Alto & 1,6 \\
Cobertura Social Odontológica & \\
Con cobertura & 56,4 \\
Sin cobertura & 43,6 \\
Percepción sobre la Salud Bucal & \\
Muy buena & 7,3 \\
Buena & 34,4 \\
Regular & 47,0 \\
Mala & 9,6 \\
Muy mala & 1,7 \\
Conocimientos de Salud Bucal & \\
La consulta periódica al odontólogo ayuda & 92,7 \\
a prevenir la caries dental & \\
La consulta periódica al odontólogo ayuda & 91,6 \\
a prevenir la inflamación de las encías & \\
Hábitos de Higiene Bucal & \\
Frecuencia diaria del cepillado dental & \\
$\quad$ No se cepilla los dientes & \\
$\quad$ Se cepilla 1 vez al día & \\
$\quad$ Se cepilla 2 veces al día & 25,2 \\
$\quad$ Utilización diaria del hilo dental & \\
\hline & \\
\hline & \\
\hline
\end{tabular}

En el área de la salud pública existen 5 hospitales, 14 centros de atención primaria de la salud (CAPS) y 37 salas de atención primaria (SAPS) que brindan prestaciones odontológicas de distinto grado de complejidad ${ }^{21,22}$.

El presente estudio muestra que el $67 \%$ de la población de estudio asistió a una consulta odontológica en el trascurso del año anterior a la entrevista, pero solo el $27 \%$ fue por motivos preventivos. Esto indica que el uso de servicios odontológicos está orientado a paliar los síntomas provocados por las patologías orales, pues la mayor parte de los individuos solo utilizan los servicios de salud ante situaciones de dolor dental y urgencias odontológicas y en menor medida para tratamientos dentales y para controles o chequeos dentales de rutina. En tal sentido, numerosas investigaciones realizadas en diversos contextos $^{23-25}$ han reportado que la búsqueda de atención odontológica ocurre principalmente por razones sintomáticas y a menudo la extracción de dientes es el servicio prestado para aliviar el dolor.

La baja proporción de encuestados que utiliza servicios de salud odontológicos por motivos preventivos, y el hecho de que la situación no se encuentre asociada a los conocimientos en salud bucal, al nivel socioeconómico y a la cobertura social odontológica podría deberse a factores no contemplados en la investigación como ser: la insatisfacción con los servicios prestados en el 
Tabla 2. Factores asociados a la utilización de Servicios de Salud Odontológicos en los últimos 12 meses. Individuos de 35 a 44 años. Ciudad de Corrientes; Argentina. 2013.

\begin{tabular}{lccc}
\hline \multicolumn{1}{c}{ Variables } & OR & IC 95\% & Valor de p \\
\hline Sexo & 1,28 & $(0,83-1,97)$ & 0,24 \\
Nivel Socioeconómico & 2,80 & $(1,59-4,91)$ & 0,00 \\
Cobertura social odontológica & 1,74 & $(1,13-2,68)$ & 0,01 \\
$\begin{array}{l}\text { Percepción sobre la salud bucal } \\
\text { Conocimiento: La consulta periódica al odontólogo puede ayudar a }\end{array}$ & 2,16 & $(1,37-3,41)$ & 0,00 \\
prevenir la caries dental & 2,18 & $(1,00-4,72)$ & 0,04 \\
$\begin{array}{l}\text { Conocimiento: La consulta periódica al odontólogo puede ayudar a } \\
\text { prevenir la inflamación de las encías. }\end{array}$ & 2,90 & $(1,39-6,00)$ & 0,00 \\
$\begin{array}{l}\text { Frecuencia diaria del cepillado dental (más de dos veces) } \\
\text { Utilización diaria del hilo dental }\end{array}$ & 3,61 & $(2,29-5,70)$ & 0,00 \\
\hline
\end{tabular}

Tabla 3. Factores asociados a la utilización de Servicios de Salud Odontológicos por chequeo dental/control de ruina. Individuos de 35 a 44 años. Ciudad de Corrientes; Argentina. 2013.

\begin{tabular}{lccc}
\hline \multicolumn{1}{c}{ Variables } & OR & IC 95\% & Valor de $\mathbf{p}$ \\
\hline Sexo & 0,78 & $(0,49-1,22)$ & 0,28 \\
Nivel Socioeconómico & 1,78 & $(0,89-3,59)$ & 0,09 \\
Cobertura social odontológica & 1,30 & $(0,83-2,04)$ & 0,24 \\
Percepción sobre la salud bucal & 2,25 & $(1,42-3,57)$ & 0,00 \\
Conocimiento: La consulta periódica al odontólogo puede ayudar a & 2,00 & $(0,74-5,42)$ & 0,16 \\
prevenir la caries dental & & & \\
Conocimiento: La consulta periódica al odontólogo puede ayudar a & 1,66 & $(0,66-4,17)$ & 0,27 \\
prevenir la inflamación de las encías. & & & \\
Frecuencia diaria del cepillado dental (más de dos veces) & 1,72 & $(1,03-2,88)$ & 0,03 \\
Utilización diaria del hilo dental & 3,09 & $(1,92-4,99)$ & 0,00 \\
\hline
\end{tabular}

sector público, los costos que implica la consulta odontológica en el sector privado, los alcances de la cobertura social odontológica o problemas personales relacionados con el miedo a la atención odontológica, la presencia de problemas de movilidad, la disponibilidad de tiempo, entre otros. En futuros estudios deberían explorarse los aspectos mencionados.

Hay autores que señalan el papel de las características socio-político y culturales de espacios geográficos como un importante factor determinante de la acumulación de secuelas orales, indicando que los individuos que viven en barrios de mayor exclusión social reportaron ser más propensos a utilizar servicios de salud dental ante situaciones de urgencias odontológicas ${ }^{26}$. En la Ciudad de Corrientes es necesario que en futuros estudios se explore la ubicación geográfica como posible factor determinante de la utilización de servicios de salud odontológicos.
El sexo no resultó ser una variable significativa en la utilización de servicios oral en lo que se refiere a la frecuencia y al uso por motivos preventivos. Esto indicaría que no existe un mayor interés por parte de mujeres y hombres por el cuidado de su apariencia personal en lo referente a la salud bucal. Estos resultados coinciden con Uysal et al. ${ }^{23}$ y con Fonseca et al. ${ }^{26}$ y difieren de otros estudios que reportaron mayor utilización de servicios de salud odontológicos en individuos de sexo femenino $3,5,23,27,28$.

El ingreso ${ }^{28}$, así como el nivel socioeconómi$\mathrm{Co}^{29}$ son variables que se han relacionado fuertemente con la utilización de los servicios de salud, debido a que representan la capacidad de pago para acceder a un servicio y constituyen una de las barreras más frecuentemente reportadas ${ }^{30}$.

En Argentina, De Santis \& Herrero $^{31}$ señalan que la utilización de los servicios de salud está concentrada en los individuos más favorecidos y 
que dicha desigualdad se debe en su mayor parte a la influencia de variables tales como el ingreso per cápita del hogar, el nivel educativo, la posesión de cobertura de salud, el lugar de residencia, entre otros. En esta investigación, un nivel socioeconómico más alto, se asoció a una mayor utilización de servicios de salud odontológicos en los últimos 12 meses, resultado similares fueron descriptos en otros trabajos ${ }^{27,29,32}$. Hay autores que reportaron que los individuos con mayor nivel de educación presentan mayor utilización de servicios de salud bucal. En tal sentido, hay que destacar que en la presente investigación la educación se consideró como un indicador que forma parte del nivel socioeconómico.

Estudios realizados en Brasil hallaron que las personas con mayores niveles de educación e ingresos presentaban mayores tasas de prevalencia de uso reciente de servicios dentales. El mayor ingreso puede facilitar la compra de un servicio y la educación superior puede aportar información sobre la importancia del uso regular de los servicios dentales ${ }^{5,28-33}$.

En lo referente a la auto-percepción de la salud bucal, los individuos que tienen una percepción buena presentaron mayores probabilidades de realizar una consulta odontológica en los últimos 12 meses anteriores a la entrevista y mayores probabilidades de haber realizado una consulta odontológica por motivos de control de rutina/ chequeos dental. Estos resultados son similares a los hallados por Machado et al. ${ }^{28}$ quienes encontraron que una de las variables más fuertemente asociada con el uso regular de los servicios dentales fue la auto-percepción de la salud oral. Las personas que reportaron como muy buena tenían una prevalencia de uso regular 4.4 veces mayor en comparación con aquellos que creían que tenían mala, muy mala salud/oral ${ }^{28}$.

En Argentina la Encuesta Nacional de factores de Riesgo, registró que un mayor nivel de cobertura de salud se asoció a mayor ingreso, mayor nivel educativo y mayor edad, tanto a nivel nacional como a nivel de cada provincia, entre ellas, la Provincia de Corrientes ${ }^{34}$. En el presente estudio la cobertura social odontológica, se asoció a una mayor probabilidad de utilización de servicios de salud odontológicos en los últimos 12 meses, pero no influyó en la consulta por motivos de prevención. Estos resultados concuerdan con Slack-Smith et al. ${ }^{27}$ quienes reportaron que en su población de estudio tener seguro médico privado se asoció a una mayor probabilidad de haber consultado al odontólogo en los últimos 12 meses. Resultados similares fueron descriptos por otros autores ${ }^{5,29,35}$.
Por otro lado, mejores hábitos de higiene bucodental se asociaron significativamente a una mayor utilización de servicios de salud odontológicos en lo referente a la frecuencia a y motivos preventivos. Estos resultados coinciden con los reportados por Medina-Solís et al. ${ }^{29}$.

Los conocimientos de salud bucodental se asociaron significativamente a la consulta odontológica en los últimos 12 meses. Pero el hecho de conocer que la consulta periódica al odontólogo puede contribuir a prevenir la caries dental y la inflamación gingival no resultaron ser variables significativas de la consulta odontológica por motivos de control/chequeo dental. Estas diferencias podrían explicarse por el hecho de que los individuos que asisten a consultas odontológicas con mayor frecuencia lo hacen para realizarse tratamientos dentales y no específicamente para un control rutinario. Estos resultados difieren de Chandra et al. ${ }^{36}$ quienes hallaron una mayor probabilidad de buscar atención dental preventiva asociado con el conocimiento de la salud dental. Los autores afirman que los motivos que provocaron a las personas buscar el cuidado dental preventivo incluyen las creencias que uno es susceptible a las enfermedades dentales, que los problemas dentales son graves, y que el tratamiento dental es beneficioso. Los que creen que son altamente susceptibles a las enfermedades dentales hacen visitas dentales más preventivas.

\section{Consideraciones generales de la metodología de trabajo}

Las encuestas constituyen un método relativamente sencillo y rápido de recogida de información, aunque presentan limitaciones y dificultades metodológicas importantes, como las referidas al diseño de la muestra que, para que sea representativa de la población, exige la utilización de diseños muestrales complejos y un elevado tamaño muestral que permita hacer generalizaciones a toda la población con un error muestral conocido y tolerable ${ }^{37}$. En el presente trabajo, la muestra observada es representativa de la población de estudio (individuos adultos de 35 a 44 años). Los resultados son generalizables; ya que se siguió con rigurosidad el procedimiento aleatorio de la selección de viviendas y se respetaron las cuotas de sexo y edad de los individuos entrevistados.

Entre las limitaciones, se puede mencionar la tendencia de los entrevistados a dar las respuestas socialmente deseables (Ej. Afirmar que asistió a la consulta odontológica por motivos preventivos). 
Otra de las limitaciones que presenta la encuesta es el sesgo del recuerdo (Ej. Recordar si la consulta odontológica se realizó en los últimos 12 meses anteriores a la entrevista). No obstante en el trabajo realizado se lograron identificar relaciones existentes entre las variables en estudio.

A pesar de las posibles limitaciones mencionadas, el presente trabajo proporciona una visión general de los factores que se asocian a la utilización de servicios de salud odontológicos en la población adulta que vive en la Ciudad de Corrientes. Hasta el momento un estudio similar no se hizo en la región. Entre sus fortalezas se destacan la conformación de una base de datos para la comparación de los resultados con estudios similares que se realicen en el futuro.

\section{Conclusión}

El estudio documenta información relevante en el campo de la salud pública que debería tenerse en cuenta en la formulación de políticas de salud dirigidas a reducir el impacto de la salud bucal en la calidad de vida de los individuos de 35 a 44 años que viven en la ciudad de Corrientes.

En atención a los resultados de la investigación, los ejes de la implementación de programas de promoción de salud y de prevención de las enfermedades bucodentales, deberían focalizarse en: implementar medidas tendientes a aumentar la utilización de servicios de salud odontológicos con fines de prevención en individuos de ambos sexos, de todos los niveles socioeconómicos, especialmente en aquellos menos favorecidos; aumentar la cobertura social odontológica de la población adulta de la Ciudad de Corrientes; enfatizar la educación sobre cuidados referentes a la salud bucodental, promoviendo un mayor conocimiento y hábitos higiénicos adecuados.

El estudio puede utilizarse como base para futuras investigaciones de base poblacional que incorporen variables no contempladas como ser la incidencia de otros factores que influyan en la utilización de servicios de salud bucal, especialmente por motivos preventivos; las barreras para no acudir a la consulta odontológica, la satisfacción de los usuarios con los servicios prestados.

\section{Agradecimientos}

Al Consejo Nacional de Investigaciones Científicas y Técnicas -CONICET- y a la Universidad Nacional del Nordeste-UNNE- por el financiamiento de la investigación. 


\section{Referencias}

1. Henostroza Haro G. Caries dental. Principios y procedimientos para el diagnóstico. Lima: Editorial Medica Ripano; 2007.

2. American Dental Association. Hábitos saludables [Internet]. [accesado 2014 Oct 2]. Disponible en: http:// www.mouthhealthy.org/en/adults-under-40/healthy -habits/

3. Masalu JR, Kikwilu EN, Kahabuka FK, Senkoro AR, Kida IA. Oral health related behaviors among adult Tanzanians: a national pathfinder survey. BMC Oral Health 2009; 9:22.

4. Carrasco-Loyola M, Sáenz AL, Orejuela-Ramírez F. Associated factors with the use of dental services in a peruvian university dental clinical. Rev Estomatol Herediana 2015; 25(1):27-35.

5. Dal-Bó Coradini MC, Peres MA. Determinantes da utilização de serviços odontológicos entre adultos: um estudo de base populacional em Florianópolis, Santa Catarina, Brasil. Cad Saude Publica 2013; 29 (11):23192332.

6. Núñez L, Icaza G, Contreras V, Correa G, Canales T, Mejía G, Oxman-Martinez J, Moreau J. Factores asociados a la consulta odontológica en niños/as y jóvenes de Talca (Chile) e inmigrantes chilenos de Montreal (Canadá). Gac Sanit 2013; 27(4):344-349.

7. Silberman M, Marín G, Pozzio M, Sanguinetti C. El rostro sin dientes del sistema de salud". Salud bucal y el sistema de salud como determinante social. Una experiencia en la provincia de Buenos Aires, Argentina. Archivos de Medicina Familiar y General 2013; 10(1):22-29.

8. Quiñonez C, Grootendorst P. Equity in dental care among Canadian households. Int J Equity Health 2011; 10:14.

9. Pizarro V, Ferrer M, Domingo-Salvany A, Benach J, Borrell C, Pont A, Schiaffino A, Almansa J, Tresserras R, Alonso J. The utilization of dental care services according to health insurance coverage in Catalonia (Spain). Community Dent Oral Epidemiol 2009; 37(1):78-84.

10. Belló M, Becerril-Montekio VM. Sistema de salud de Argentina. Salud pública Méx 2011; 53(2):96-109.

11. Programa de las Naciones Unidas para el Desarrollo. Informe Nacional sobre Desarrollo Humano 2013 Argentina en un mundo incierto: Asegurar el desarrollo humano en el siglo XXI. 2013. [Internet]. [accesado $2015 \mathrm{Jul}$ 20]. Disponible en: http://www.ar.undp.org/content/ dam/argentina/Publications/Desarrollo\%20Humano/ AR Gentina-PNUD-INDH\%202013.pdf

12. Oral Health Surveys. Basic Methods. World Health Organization. $5^{\text {th }}$ ed. 2013. [Internet]. [accesado 2015 Dic 1]. Disponible en: http://www.icd.org/content/publications/WHO-Oral-Health-Surveys-Basic-Methods5thEdition-2013.pdf

13. Instituto Nacional de Estadística y Censo. Cuadro P2-D. Provincia de Corrientes, departamento Capital. Población total por sexo e indice de masculinidad, según edad en años simples y grupos quinquenales de edad. Año 2010. [Internet]. [accesado 2012 Jul 4]. Disponible en: http://www.censo2010.indec.gov.ar/CuadrosDefinitivos/P2- D_18_21.pdf
14. Perez KG, Peres MA, Demarco FF, Tarquínio SB, Horta BL, Gigante DP. Oral health studies in the 1982 Pelotas (Brazil) birth cohort: methodology and principal results at 15 and 24 years of age. Cad Saude Publica 2011; 27(8):1569-1580.

15. Dhó MS, Vila VG, Palladino AC. Situación de salud bucal de pacientes mayores de 18 años. Cátedra Práctica Clínica Preventiva I, Facultad de Odontología UNNE, Argentina, 2010. Rev Fac Odontol Univ Antioq 2013; 24(2):214-231.

16. Campo-Arias A, Oviedo HC. Propiedades psicométricas de una escala: la consistencia interna. Rev. salud pública 2008; 10(5):831-839.

17. Organización Panamericana de la Salud. Ministerio de Salud Pública Presidencia de la Nación. Indicadores Básicos Argentina 2012. [Internet]. [accesado $2015 \mathrm{Jul}$ 20]. Disponible en: http://www.msal.gov.ar/images/ stories/pdf/indicadores-basicos-2012.pdf

18. Ministerio de Salud Pública de la Provincia de Corrientes. [Internet]. [accesado 2015 Sept 18]. Disponible en: http://www.saludcorrientes.gob.ar/s/desc/ sala/2015/1/3/personas_con_cobertura_social _y_sin_ cobertura.jpg.jpg

19. Buffil CE, López Vallejos MJ, Cardozo ME. Causas más frecuentes que provocan la extracción dentaria en la población de Corrientes. Rev. de la Facultad de Odontología UNNE 2009; II(3):20-24.

20. Chetti AM, Ruiz Díaz EO, Romero HJ. Estudio de la prevalencia de caries en piezas dentarias anteriores y su relación con variables epidemiológicas. UNNE, Comunicaciones Científicas y Tecnológicas 2005; M-17.

21. Ministerio de Salud Pública de la Provincia de Corrientes. [Internet]. [accesado 2015 Sept 18]. Disponible en: http://saludcorrientes.gob.ar/sitio/index.php/el-ministerio/misiones-yfunciones/telefonos-utiles/capital/ caps-capital.html

22. Municipalidad de la Ciudad de Corrientes. Salas de Atención Primaria. [Internet]. [accesado 2015 Sept 18]. Disponible en: http://ciudaddecorrientes.gov.ar/corrientes/saps

23. Uysal S, Alev F, Yamalik N, Etikan I. Unmet Need for Education by Professionals and Inconsistency in Knowledge and Behavior of Dental Patients. NY State Dent J 2010; 76(3):16-21

24. Jamjoum H. Preventive oral health knowledge, practice and behaviour in Jeddah, Saudi Arabia. Odonto-Stomatologie Tropicale p. 13-18. [Internet] [accesado 2015 Sept 15]. Disponible en: http://www.santetropicale.com/resume/48004.pdf

25. Lian CW, Phing TS, Chan CS, Shin BC, Baharuddin LH, Che'Jalil ZB. Oral health knowledge, attitude and practice among secondary school students in Kuching, Sarawak. Arch Oro facial Sciences 2010; 5(1):9-16.

26. Fonseca DAV, Mialhe FL, Ambrosano GM, Pereira AC Meneghim MC. Influence of the organization of primary care and the socio-demographic characteristics of the population on the demand for municipal emergency dental care. Cien Saude Colet 2014; 19(1):269278. 
27. Slack-Smith LM, Mills CR, Bulsara MK, O'Grady MJ. Demographic, health and lifestyle factors associated with dental service attendance by young adults. Aust Dent J 2007; 52(3):205-209.

28. Machado LP, Camargo MB, Milanez JCJ, Bastos GAN. Uso regular de serviços odontológicos entre adultos e idosos em região vulnerável no sul do Brasil. Rev Saude Publica 2012; 46(3):526-533.

29. Medina-Solís CE, Villalobos-Rodelo JJ, Márquez-Corona ML, Vallejos-Sánchez AA, Portillo-Núñez CL, Casanova-Rosado AJ. Desigualdades socioeconómicas en la utilización de servicios de salud bucal: estudio en escolares mexicanos de 6 a 12 años de edad. Cad Saude Publica 2009; 25(12):2621-2631.

30. Mariño RJ, Khan AR, Tham R, Khew CW, Stevenson C. Pattern and factors associated with utilization of dental services among older adults in rural Victoria. Aust Dent J 2014; 59(4):504-510.

31. De Santis M, Herrero V. Equidad en el Acceso, Desigualdad y Utilización de los Servicios de Salud Una Aplicación al Caso Argentino en 2001. Revista de Economía y Estadística 2009; XLVII(1):125-126

32. Rocha-Buelvas A. Análise sobre o acesso a serviços da saúde bucal: um indicador de equidade. Rev. Gerenc. Polit. Salud 2013; 12(25):96-112.

33. Ferreira CO, Ferreira JL, Bof de Andrade F. Fatores associados a utilizacao dos servicos odontologicos por idosos brasileiros. Rev Saude Publica 2013; 47(3):90-97.

34. Ministerio de Salud Argentina. Segunda Encuesta Nacional de factores de Riesgo para enfermedades no transmisibles. [Internet] [accesado 2015 Sept 18] Disponible en: http://www.msal.gob.ar/ent/images/stories/vigilancia/pdf/fr_encuestanacional-factores-riesgo-2011.pdf

35. Delgado B, Cornejo-ovalle M, Jadue HL, Huberman J. Determinantes sociales yequidad de acceso en la salud dental en Chile. Cient. dent. 2013; 10(2):101-109.

36. Chandra Shekar BR, Reddy C, Manjunath BC, Suma S. Dental health awareness, attitude, oral health-related habits, and behaviors in relation to socio-economic factors among the municipal employees of Mysore city. Ann Trop Med Public Health 2011; 4:99-106

37. D’Ancona MAC. Métodos de encuestas. Teoría y Práctica, Errores y Mejoras. Madrid: Ed. Síntesis; 2004.

Artigo apresentado em 29/06/2015

Aprovado em 20/01/2016

Versão final apresentada em 22/01/2016 